\title{
Rapid Brain Nicotine Uptake from Electronic Cigarettes
}

\author{
Kiran Kumar Solingapuram Sai*1, Yantao Zuo*2, Jed E. Rose ${ }^{2}$, Pradeep K. Garg ${ }^{1}$, Sudha Garg ${ }^{1}$, Rachid Nazih ${ }^{1}$, \\ Akiva Mintz ${ }^{\dagger 1}$, and Alexey G. Mukhin ${ }^{\dagger 2}$ \\ ${ }^{I}$ Department of Radiological Sciences, Wake Forest Baptist Medical Center, Winston-Salem, North Carolina; and ${ }^{2}$ Department of \\ Psychiatry and Behavioral Sciences, Duke University Medical Center, Durham, North Carolina
}

This study sought to determine brain nicotine kinetics from use of the increasingly popular electronic cigarette (E-cig). Methods: In $17 \mathrm{E}$-cig users (9 men and 8 women), brain uptake of nicotine after inhalation from E-cigs was directly assessed using ${ }^{11} \mathrm{C}$-nicotine PET. The brain nicotine kinetics were compared with those from smoking combustible cigarettes (C-cigs). Results: A single puff of E-cig vapor caused the nicotine concentration in the brain to rise quickly (mean time to reach $50 \%$ of maximum brain nicotine concentration, $27 \mathrm{~s}$ ), with a peak amplitude $25 \%$ higher in women than men, resembling previous observations with C-cigs. Nonetheless, the accumulation from E-cigs $(24 \%)$ was less than that from C-cigs $(32 \%)$ in both men and women. Conclusion: E-cigs can deliver nicotine to the brain with a rapidity similar to that of $\mathrm{C}$-cigs. Therefore, to the extent that rapid brain uptake promotes smoking reward, E-cigs might maintain a degree of nicotine dependence and also serve as a noncombustible substitute for cigarettes.

Key Words: nicotine; electronic cigarettes; e-cigarettes; ENDS; smoking; vaping

J Nucl Med 2020; 61:928-930

DOI: 10.2967/jnumed.119.230748

$\mathbf{R}$ ecently, there has been enormous growth in the popularity of electronic cigarettes (E-cigs) (1,2). Although E-cigs are likely less harmful than combustible cigarettes (C-cigs), a concern is that use of these products can lead to the development and maintenance of nicotine dependence. As with other abused drugs, the rate and magnitude of nicotine accumulation in the brain may contribute strongly to its acute reinforcing effects (3-9). The proposed continuum of nicotine-containing products' abuse liability (10) is quite close to the continuum of the rapidity of nicotine delivery to the brain (highest for cigarettes and lowest for nicotine patches). In several recent studies (11-13) performed on experienced E-cig users, increases in nicotine concentration in the venous blood after E-cig use were comparable to those after C-cig smoking. Venous concentrations, however, do not accurately reflect brain levels; the capability of E-cigs to produce fast nicotine delivery to the brain, as previously observed with C-cigs $(14,15)$, has not been studied.

Received Jul. 22, 2019; revision accepted Oct. 9, 2019.

For correspondence or reprints contact: Alexey G. Mukhin, Duke University Medical Center, 2424 Erwin Rd., Suite 201, Mailcode 2701, Durham, NC 27705.

E-mail: a.mukhin@duke.edu

${ }^{*}$ Contributed equally to this work.

tContributed equally to this work

Published online Nov. 1, 2019.

COPYRIGHT (C) 2020 by the Society of Nuclear Medicine and Molecular Imaging.
Here, we report the results of the first—-to our knowledge-direct assessment of brain uptake of nicotine from E-cig use. Sex differences were also examined, in view of the previously observed sex differences in nicotine accumulation in the brain with C-cigs (16).

\section{MATERIALS AND METHODS}

PET scanning after inhalation of E-cig vapor was conducted on $17 \mathrm{E}-$ cig users, 3 of whom were also scanned after they inhaled C-cig smoke at a separate session. For comparison, PET data from $19 \mathrm{C}$-cig smokers who completed a previously reported study (16) were also included for the present analysis. Participants in each group were recruited from the Winston-Salem, NC, area. The inclusion criteria were an age of 18-65 y, general good health, and use of E-cigs at least 4 times per month or Ccigs at least 8 times per day. The exclusion criteria included respiratory or cardiovascular diseases, psychiatric disorders, alcohol abuse, illicit drug use, and contraindications to PET scanning (e.g., pregnancy). The E-cig group included 8 current smokers, 8 former smokers, and 1 person who had never smoked. The E-cig and C-cig groups were comparable in sex composition (male/female, $9 / 8$ vs. $9 / 10$, respectively), racial composition (Caucasian/African-American/others, $70.6 \% / 17.6 \% / 5.9 \%$ vs. $73.7 \% / 26.3 \% / 0$, respectively) ( $\chi^{2}$ tests, not statistically significant), mean age $\pm \mathrm{SD}(43 \pm 13$ vs. $44 \pm 10 \mathrm{y}$, respectively), mean body weight $\pm \mathrm{SD}(87 \pm 16 \mathrm{vs} .82 \pm 19 \mathrm{~kg}$, respectively), and mean years of smoking $\pm \mathrm{SD}(21 \pm 14$ vs. $24 \pm 12$, respectively) ( $t$ tests, not statistically significant). The institutional review boards of the Duke University Health System and of Wake Forest University Health Sciences approved this study, and all subjects gave written informed consent.

After inhaling a single puff of vapor or smoke containing ${ }^{11} \mathrm{C}$ nicotine, each participant underwent PET scanning of the head. A standardized puff of vapor was produced from $15 \mu \mathrm{L}$ of V2 Red e-liquid (1.2\% nicotine, 20/80 vegetable glycerin/propylene glycol; Mig Vapor LLC) mixed with ${ }^{11} \mathrm{C}$-nicotine via a V2 EX Blanks refillable cartomizer (Mig Vapor LLC) coupled with a programmable air syringe pump. The smoke was generated from a shortened Basic Gold 100s hard-pack cigarette (Philip Morris USA) through a customized smoke delivery device after ${ }^{11} \mathrm{C}$-nicotine was applied $(16,17)$. The subject's head was scanned for just over $12 \mathrm{~min}$ in a sequence of 245 frames of 1-4 s each. Afterward, a full-body scan was conducted to measure the total absorbed dose of ${ }^{11} \mathrm{C}$-nicotine, which was used to normalize the ${ }^{11} \mathrm{C}$ nicotine uptake values between subjects and between conditions. ${ }^{11} \mathrm{C}$ nicotine was synthesized following an established protocol (18). The PET scans were conducted using a Discovery MI DR PET/CT scanner (GE Healthcare). PET images were processed using PMOD.

Whole-brain ${ }^{11} \mathrm{C}$-nicotine radioactivity over time was calculated as a percentage of total absorbed dose per kilogram of brain tissue. After the individual brain time-activity curves were subjected to 3-exponential curve fitting (11), kinetic parameters were calculated (i.e., maximum brain nicotine concentration $\left[\mathrm{C}_{\max }\right]$, area under the brain time-activity curve [AUC], and time to reach $50 \%$ of $\mathrm{C}_{\max }\left[\mathrm{T}_{1 / 2}\right]$ ). ANOVA with 2 
between-subject factors (E-cig vs. C-cig; sex) was conducted on each of the 3 parameters. Additional analyses of covariance with years of smoking or body weight, each entered as a covariate, were also performed, given the possible effects of either variable on nicotine kinetics. The threshold for statistical significance was set at a $P$ value of less than 0.05 (2-tailed). Group mean values $( \pm$ SEM) are reported unless otherwise specified.

\section{RESULTS}

The average ${ }^{11} \mathrm{C}$-nicotine activity curves for the brain are shown in Figures 1A and 1B. Both product group and sex were significant factors affecting $\mathrm{C}_{\max }$ (Fig. 1C) and AUC (Fig. 1D). Mean $\mathrm{C}_{\max }$ was $30.4 \%$ lower in the E-cig group than in the C-cig group $(24.2 \%$ in men and $32.3 \%$ in women), and mean AUC was $28.9 \%$ lower (24.7\% in men and $30.2 \%$ in women).

After E-cig vapor inhalation, $\mathrm{C}_{\max }$ was $24.6 \%$ greater in women than in men, and AUC was $25.3 \%$ greater in women than in menfindings that are similar to our observation with C-cigs $(32.7 \%$ and $31.6 \%$ greater, respectively). Mean $\mathrm{T}_{1 / 2}$ did not significantly differ between the 2 products. A trend for a shorter $\mathrm{T}_{1 / 2}$ in women than in men was observed $(22.5 \pm 5.0 \mathrm{~s}$ vs. $30.8 \pm 5.4 \mathrm{~s}$ for E-cig; $19.1 \pm$ 2.2 s vs. $27.8 \pm 4.8$ s for C-cig; $P=0.065$ ). No product $\times$ sex interaction was found for any of the 3 kinetic parameters.

The differences in brain nicotine kinetics between these 2 products and between sexes remained statistically robust in additional analyses of covariance with either body weight or years of smoking entered as a covariate.

A comparison of the whole-body distribution of the radioactivity after inhalation from E-cigs or C-cigs revealed higher oropharyngeal or tracheobronchial deposition of nicotine after E-cig use. To illustrate
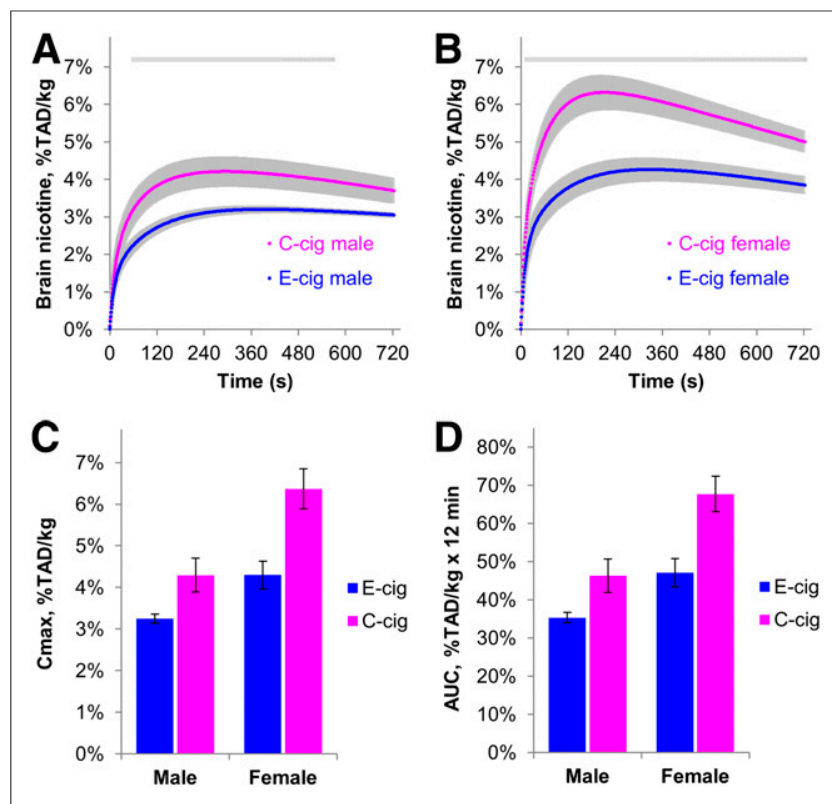

FIGURE 1. Curves and kinetic parameters for nicotine accumulation in brain after single puff of E-cig vapor (mean \pm SEM, 9 men and 8 women) or ${ }^{11} \mathrm{C}$-nicotine-containing $\mathrm{C}$-cig smoke (9 men and 10 women). Gray lines represent time interval when difference between products was statistically significant ( $t$ test, $P<0.05$ ). Mean $\mathrm{C}_{\max }$ and AUC differed between products ( $P=0.0002$ and 0.0003 , respectively) and sexes ( $P=0.0002$ and 0.0001 , respectively) but without interactions of these 2 factors. Nicotine accumulation per kilogram of brain mass is expressed as percentage of total absorbed dose (TAD) of ${ }^{11} \mathrm{C}$-nicotine. this observation, we performed 2 whole-body PET scans on 3 participants, each inhaling E-cig vapor and C-cig smoke in separate sessions. A representative result is shown in Figure 2.

\section{DISCUSSION}

This study produced 3 new and important findings: first, that E-cigs can deliver nicotine to the brain with a rapidity similar to that of $\mathrm{C}$ cigs; second, that the magnitude of brain nicotine accumulation from E-cigs in both men and women was about $30 \%$ less than that from Ccigs; and third, that the magnitude of brain nicotine accumulation after E-cig use in women is about $24 \%$ higher than that in men, resembling the sex difference previously reported for C-cigs.

After inhalation of a single puff of E-cig vapor, the nicotine concentration in the brain rose quickly, similar to that after a puff from C-cigs (mean $\mathrm{T}_{1 / 2}, 27 \pm 4 \mathrm{~s}$ and $23 \pm 3 \mathrm{~s}$, respectively). This temporal profile suggests that the primary route of nicotine delivery to the blood after E-cig use is alveolar absorption, leading to the rapid rise of nicotine concentration in arterial blood. Nonetheless, $\mathrm{C}_{\max }$ and AUC for E-cigs were two thirds those for C-cigs. Since nicotine uptake by the brain is dependent on regional cerebral blood flow (19-21), these differences could be explained by a slower brain blood flow after use of E-cigs or a lower nicotine concentration in the arterial blood. Both of these possible explanations remain to be verified in future studies. However, our preliminary results suggest that the lower brain nicotine accumulation after E-cig use than after C-cig use may be due in part to a lower arterial-blood nicotine concentration, which would be due to greater nicotine retention in the upper respiratory tract from vapor than from smoke. The effect of such retention is that less nicotine

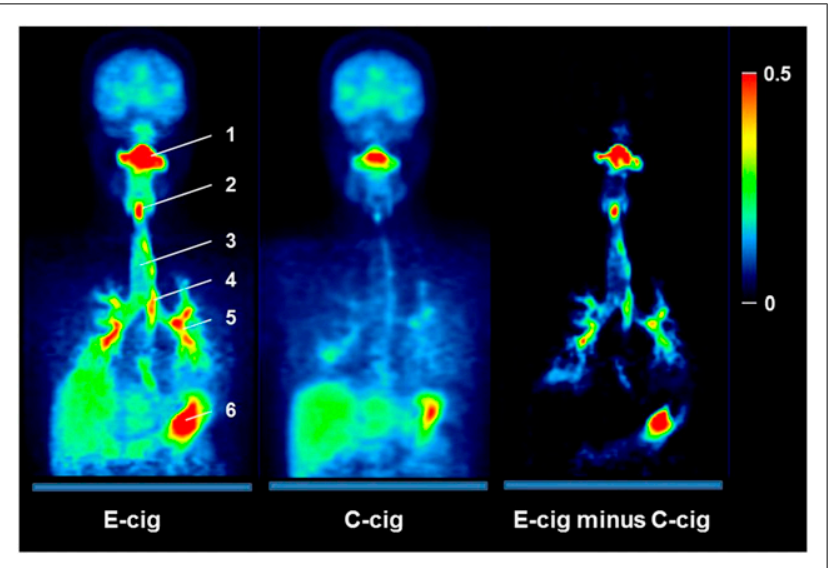

FIGURE 2. Oropharyngeal and tracheobronchial deposition of nicotine after use of E-cig and C-cig. Images show sum of coronal slices of 3dimensional radioactivity distribution assessed at $18 \mathrm{~min}$ after inhalation of single puff from respective ${ }^{11} \mathrm{C}$-nicotine-containing product and expressed as percentage of total absorbed dose per kilogram of tissue. Maximum value of pseudo color scale is $0.5 \%$ total absorbed dose $/ \mathrm{cm}^{2}$. Subtraction of C-cig image from E-cig image shows higher retention of nicotine in the oropharyngeal, tracheobronchial, and stomach areas after E-cig versus C-cig use. Subtraction of E-cig image from C-cig image (not shown) did not show specific places where C-cig produced visibly greater nicotine concentration than E-cig; rather, there was slight increase in nicotine concentration throughout body outside respiratory tract for C-cig condition. 1 = mouth cavity; 2 = vocal cords; 3 = trachea; 4 = esophagus; 5 = bronchi; $6=$ stomach. 
would reach the alveoli, where rapid absorption occurs. A potential explanation for this possibility is that the typically more alkaline $\mathrm{pH}$ of E-cig liquids than of C-cig smoke ( $\mathrm{pH} 7-9$ for E-cigs and 5-6 for C-cigs $(22,23)$ ) enhances evaporation of nicotine base from droplets and its retention in the respiratory tract. Such deposition of nicotine is likely to be reduced by using an E-cig liquid with a low $\mathrm{pH}$.

The observed more intensive brain nicotine accumulation from E-cigs in women than men might reflect sex differences in respiratory tract anatomy (16) or in hemodynamics. Since the accumulation of nicotine in the brain depends on blood flow (19-21), the higher accumulation in women might be explained by the approximately $35 \%$ higher ratio of cerebral blood flow to cardiac output in women than in men (24).

Note that the slower brain nicotine delivery of E-cigs than of C-cigs can be compensated for by the higher nicotine content of e-liquids or by more intensive vaping to achieve a desired effect.

\section{CONCLUSION}

Our results suggest that E-cigs can deliver nicotine to the brain with a rapidity similar to that of $\mathrm{C}$-cigs and that there is a sex difference in this delivery. Therefore, to the extent that rapid brain uptake promotes smoking reward, E-cigs might maintain a degree of nicotine dependence and also serve as a noncombustible substitute for cigarettes.

\section{DISCLOSURE}

This research was supported by the NIH (R01 DA044756, R03 DA029676, P30 CA012197-35, UL1 TR001420, and UL1 TR001873) and the American Cancer Society (124443-MRSG-13-121-01-CDD). Jed Rose receives grants from JUUL Labs Inc.; grants, personal fees, and a patent purchase agreement on a nicotine delivery system with Philip Morris International; grants from Altria; grants and personal fees from Intratab Labs Inc.; grants from the National Institute on Drug Abuse; and personal fees from Embera Neurotherapeutics, outside the submitted work. In addition, Jed Rose has a patent on a licensed nicotine delivery system. No other potential conflict of interest relevant to this article was reported.

\section{ACKNOWLEDGMENTS}

We thank Sandra Norona, Allison Fulp, Jonathan Richardson, and Janiece Morgan for assistance in data acquisition and manuscript preparation.

\section{KEY POINTS}

QUESTION: What are the brain nicotine kinetics from the use of the increasingly popular E-cig?

PERTINENT FINDINGS: E-cigs delivered nicotine to the brain with a rapidity similar to that of $\mathrm{C}$-cigs. Nonetheless, the accumulation of nicotine in the brain from E-cigs was less than that from $\mathrm{C}$-cigs in both men and women.

IMPLICATIONS FOR PATIENT CARE: To the extent that rapid brain uptake promotes smoking reward, E-cigs might maintain a degree of nicotine dependence and also serve as noncombustible substitutes for cigarettes.

\section{REFERENCES}

1. Bao W, Xu G, Lu J, Snetselaar LG, Wallace RB. Changes in electronic cigarette use among adults in the United States, 2014-2016. JAMA. 2018;319: 2039-2041.

2. Mirbolouk M, Charkhchi P, Kianoush S, et al. Prevalence and distribution of Ecigarette use among U.S. adults: Behavioral Risk Factor Surveillance System, 2016. Ann Intern Med. 2018;169:429-438.

3. Benowitz NL. Clinical pharmacology of inhaled drugs of abuse: implications in understanding nicotine dependence. NIDA Res Monogr. 1990;99:12-29.

4. Henningfield JE, Keegan RM. Nicotine delivery kinetics and abuse liability. J Consult Clin Psychol. 1993;61:743-750.

5. Schneider NG, Olmstead RE, Franzon MA, Lunell E. The nicotine inhaler: clinical pharmacokinetics and comparison with other nicotine treatments. Clin Pharmacokinet. 2001;40:661-684.

6. Caldwell B, Sumner W, Crane J. A systematic review of nicotine by inhalation: is there a role for the inhaled route? Nicotine Tob Res. 2012;14:11271139 .

7. Samaha AN, Yau WY, Yang P, Robinson TE. Rapid delivery of nicotine promotes behavioral sensitization and alters its neurobiological impact. Biol Psychiatry. 2005;57:351-360.

8. Wing VC, Shoaib M. Effect of infusion rate on intravenous nicotine self-administration in rats. Behav Pharmacol. 2013;24:517-522.

9. Allain F, Minogianis EA, Roberts DCS, Samaha AN. How fast and how often: the pharmacokinetics of drug use are decisive in addiction. Neurosci Biobehav Rev. 2015;56:166-179.

10. Fagerström K, Eissenberg T. Dependence on tobacco and nicotine products: a case for product-specific assessment. Nicotine Tob Res. 2012;14:1382-1390.

11. Vansickel AR, Eissenberg TE. Electronic cigarettes: effective nicotine delivery after acute administration. Nicotine Tob Res. 2013;15:267-270.

12. Farsalinos KE, Spyrou A, Stefopoulos C, et al. Nicotine absorption from electronic cigarette use: comparison between experienced consumers (vapers) and naïve users (smokers). Sci Rep. 2015;5:11269.

13. St Helen G, Havel C, Dempsey DA, Jacob P III, Benowitz NL. Nicotine delivery, retention and pharmacokinetics from various electronic cigarettes. Addiction. 2016;111:535-544.

14. Rose JE, Mukhin AG, Lokitz SJ, et al. Kinetics of brain nicotine accumulation in dependent and nondependent smokers assessed with PET and cigarettes containing ${ }^{11}$ C-nicotine. Proc Natl Acad Sci USA. 2010;107:5190_ 5195

15. Berridge MS, Apana SM, Nagano KK, Berridge CE, Leisure GP, Boswell MV. Smoking produces rapid rise of $\left[{ }^{11} \mathrm{C}\right]$ nicotine in human brain. Psychopharmacology (Berlin). 2010;209:383-394.

16. Zuo Y, Mukhin AG, Garg S, et al. Sex-specific effects of cigarette mentholation on brain nicotine accumulation and smoking behavior. Neuropsychopharmacology. 2015;40:884-892.

17. Zuo Y, Garg PK, Nazih R, et al. A programmable smoke delivery device for PET imaging with cigarettes containing ${ }^{11} \mathrm{C}$-nicotine. J Neurosci Methods. 2017;283:55-61.

18. Halldin C, Någren K, Swahn CG, Långström B, Nybäck H. (S)- and (R) $\left[{ }^{11} \mathrm{C}\right]$ nicotine and the metabolite $(\mathrm{R} / \mathrm{S})-\left[{ }^{11} \mathrm{C}\right]$ cotinine: preparation, metabolite studies and in vivo distribution in the human brain using PET. Int J Rad Appl Instrum B. 1992;19:871880 .

19. Yokoi F, Komiyama T, Ito T, Hayashi T, Lio M, Hara T. Application of carbon-11 labelled nicotine in the measurement of human cerebral blood flow and other physiological parameters. Eur J Nucl Med. 1993;20:46-52.

20. Muzic RF Jr, Berridge MS, Friedland RP, Zhu N, Nelson AD. PET quantification of specific binding of carbon-11-nicotine in human brain. J Nucl Med. 1998;39: 2048-2054.

21. Nybäck H, Halldin C, Ahlin A, Curvall M, Eriksson L. PET studies of the uptake of $(\mathrm{S})$ - and $(\mathrm{R})-\left[{ }^{11} \mathrm{C}\right]$ nicotine in the human brain: difficulties in visualizing specific receptor binding in vivo. Psychopharmacology (Berlin). 1994;115:31-36.

22. Stepanov I, Fujioka N. Bringing attention to e-cigarette $\mathrm{pH}$ as an important element for research and regulation. Tob Control. 2015;24:413-414.

23. Armitage AK, Dixon M, Frost BE, Mariner DC, Sinclair NM. The effect of tobacco blend additives on the retention of nicotine and solanesol in the human respiratory tract and on subsequent plasma nicotine concentrations during cigarette smoking. Chem Res Toxicol. 2004;17:537-544.

24. Xing C-Y, Tarumi T, Liu J, et al. Distribution of cardiac output to the brain across the adult lifespan. J Cereb Blood Flow Metab. 2017;37:2848-2856. 